Dilean Elfy Ayaviri. ${ }^{1}$ (D) Sarali Orellana Arnez. ${ }^{2}$ Jhasmani Arispe Gutierrez. $^{3}$

Andrea Sonia Salinas Gil. $^{3}$

Maria Lorena Orellana Aguilar. $^{4}$

Correspondencia a:

${ }^{1}$ Médico Cirujano.Docente de Histología Humana, Farmacología y Terapéutica. Centro de Investigación de Salud Pública UNITEPC. "CISPU". Cochabamba - Bolivia.

${ }^{2}$ Médico Cirujano. Docente de básicas y preclínica Universidad Técnica Privada Cosmos UNITEPC. Centro de Investigación de Salud Pública UNITEPC. "CISPU". Cochabamba - Bolivia.

${ }^{3}$ Médico Cirujano.

Docente de Anatomía Humana Universidad Técnica Privada Cosmos UNITEPC

Cochabamba - Bolivia.

${ }^{4}$ Médico Cirujano.

Licenciada en Nutrición y

Dietética. Centro de Investigación de Salud Pública UNITEPC. "CISPU".

Cochabamba - Bolivia.

Email de contacto:

naelidayaviri@gmail.com airam720@hotmail.com

Procedencia y arbitraje: No comisionado, sometido a arbitraje externo

Recibido para publicación: 28 de enero del 2019 Aceptado para publicación 15 de marzo del 2019

Citar como:

Ayaviri DE, Orellana Arnez S, Arispe Gutierrez J, Salinas Gil AS, Orellana Aguilar ML. Cardioversión eléctrica y cardioversión farmacológica en pacientes con fibrilación auricular. Re Ci Sa UNITEPC. 2020; 7(1):32-42

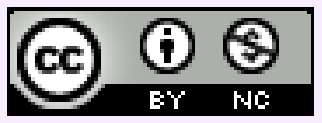

Esta obra está bajo una Licencia Creative Commons Atribución-NoComercial 4.0 Internacional.

\section{Cardioversión eléctrica y cardioversión farmacológica en pacientes con fibrilación auricular}

\section{Electrical cardioversion and pharmacological cardioversion in patients with atrial fibrillation \\ Cardioversão elétrica e cardioversão farmacológica em pacientes com fibrilação atrial}

\section{Resumen}

La fibrilación auricular altera la función hemodinámica del corazón, siendo un factor de riesgo de accidentes tromboembólicos, complicaciones y síntomas relacionados con la propia arritmia cardiaca. Restituir o conservar el ritmo sinusal es una parte importante en su manejo, para ello se establece dos tipos de Restitución, mediante una descarga eléctrica y mediante el uso de fármacos, las cuales son válidas, sin embargo existe aspectos importantes a considerar. El objetivo de este trabajo es comparar las características e indicaciones del empleo de cardioversión eléctrica y farmacológica en pacientes con fibrilación auricular. Se realizó una comparación entre ambas considerando características propias de cada método, técnicas, mecanismo, puntos importantes a tomar en cuenta, indicaciones, complicaciones y efectividad. La elección idónea del paciente y la técnica adecuada es significativo para restaurar un ritmo cardiaco normal. Ambas presentan características específicas y limitaciones. Al momento de actuar no hay procedimiento que pueda considerarse óptimo es necesario en algunas veces recurrir a una estrategia combinada se ha revisado que la prescripción de medicamentos antiarrítmicos antes de una cardioversión eléctrica puede ser útil para aumentar el éxito y prevenir.

Palabras Claves: Fibrilación Atrial, Cardioversión Eléctrica, Antiarrítmicos, Tromboembolia, Insuficiencia Cardiaca.

\section{Summary}

Atrial fibrillation alters the heart's hemodynamic function, being it a risk factor for thromboembolic accidents, complications and symptoms related with oneself cardiac arrhythmia. Restoring or preserving sinus rhythm is an important part of its management, for that two types of Restitution are established, one by an electric shock and other by the use of drugs, both are valid, however there are important aspects to consider. The work objective is to compare the characteristics and indications for the use of electrical and pharmacological cardioversion in patients with atrial fibrillation. A comparison was made between both considering the characteristics of each method, techniques, mechanism, important points to take into account, indications, complications 
and effectiveness. The right patient choice and a proper technique is significant to restore a normal heart rhythm. Both have specific characteristics and limitations. At the acting moment, there is no procedure that can be considered optimal, sometimes it is necessary to resort to a combined strategy. It has been noticed that the prescription of antiarrhythmic drugs before an electrical cardioversion can be useful to increase success and prevent it.

Keywords: Atrial Fibrillation, Electric Countershock, Anti-Arrhythmia Agents, Thromboembolism, Heart Failure.

Resumo

A fibrilação atrial altera a função hemodinâmica do coração, sendo um fator de risco para acidentes tromboembólicos, complicações e sintomas relacionados à própria arritmia cardíaca. A restauração ou preservação do ritmo sinusal é parte importante de seu manejo, sendo estabelecidos dois tipos de restituição, por meio de choque elétrico e uso de drogas válidas, porém há aspectos importantes a serem considerados. O objetivo deste trabalho é comparar as características e indicações para o uso da cardioversão elétrica e farmacológica em pacientes com fibrilação atrial. Foi feita uma comparação entre os dois considerando as características de cada método, técnicas, mecanismo, pontos importantes a serem levados em consideração, indicações, complicações e efetividade. A escolha correta do paciente e a técnica adequada são significativas para restaurar o ritmo cardíaco normal. Ambos têm características e limitações específicas. No momento da atuação, não existe procedimento que possa ser considerado ideal, às vezes é necessário recorrer a uma estratégia combinada. Revisou se que a prescrição de medicamentos antiarrítmicos antes da cardioversão elétrica pode ser útil para aumentar o sucesso e impedir.

Palavras chave: Fibrilação Atrial, Cardioversão Elétrica, Antiarrítmicos, Tromboembolia, Insuficiência Cardíaca.

\section{Introducción}

La fibrilación auricular es un ritmo irregular y rápido que consiste en la activación de la aurícula de manera descoordinada sin contracción efectiva, afectando así la función hemodinámica del corazón y siendo un factor de riesgo de accidentes tromboembólicos, complicaciones de insuficiencias cardiacas además de producir síntomas relacionados con la propia arritmia cardiaca (1-3).

La fibrilación auricular no solo incrementa el riesgo de presentar embolismo arterial y de forma específica ictus, sino que los ictus asociados suelen ser más severos $(4,5)$. Su prevalencia es de 1,5-2\% en la población general, y es previsible que en el año 2030 sea del $3 \%$ en adultos de 20 años o más (6).

Recuperar y conservar el ritmo sinusal es una parte integral del tratamiento de la fibrilación auricular. La cardioversión es un método para revertir un ritmo cardiaco anormal a un ritmo sinusal que se puede realizar mediante descarga eléctrica cardioversión eléctrica CVE o bien con fármacos cardioversión farmacológica CVF (7).

La cardioversión eléctrica y farmacológica, pueden restituir el ritmo sinusal controlando la frecuencia y el ritmo cardiaco en paciente con fibrilación auricular, ambos son opciones valederos para conseguir una reversión sinusal por tal motivo es necesario 
revisar de manera continua las recomendaciones y caracterizar las indicaciones, ventajas y procedimientos efectuados que nos permita conocer mejor la efectividad de diferentes procedimientos terapéuticos en los pacientes.

Por otro lado la fibrilación auricular, es una arritmia muy frecuente pues la mayoría de los cardiópatas la presentan en algún momento de su evolución siendo al mismo importante porque es predisponente de trombos y embolismos (8-11).

Existe una diferencia notoria en la presencia de accidente cerebro vascular, insuficiencia cardiaca y mortalidad en pacientes con fibrilación auricular versus a los pacientes sin esta enfermedad (12).

La fibrilación auricular se asocia con un elevado riesgo de complicaciones. Las tres más importantes son tromboembolismo, miocardiopatía por frecuencia ventricular rápida (taquimiocardiopatía) y muerte súbita (13).

En vista de las consecuencias hemodinámica y tromboembólicos, la conversión a ritmo sinusal puede esperarse que reduzca o suprima los síntomas y morbimortalidad asociada con la fibrilación auricular $(10,14)$.

Por todo lo sobredicho el presente trabajo permitirá mostrar las características de la cardioversión eléctrica y cardioversión farmacológica, las indicaciones en su uso y distinguir las particularidades de cada uno en el empleo en pacientes con fibrilación Auricular.

\section{Control de ritmo y cardioversión}

El control del ritmo puede ser necesario en pacientes con fibrilación auricular paroxística o persistente con el objetivo de eliminar los síntomas, mejorar la capacidad de ejercicio o prevenir la miocardiopatía inducida por taquicardia (taquimiocardiopatía) secundaria a la fibrilación auricular (15).

Según el concepto de control del ritmo cardiaco se entiende el intento de mantener o restituir el ritmo sinusal. La cardioversión es un procedimiento médico ampliamente difundido cuyo objetivo es restaurar un ritmo cardiaco anormal y rápido a un ritmo sinusal siendo una parte integral del tratamiento.

Existen dos situaciones clínicas, una de ellas es la conversión de un paciente que esta con fibrilación auricular a ritmo sinusal, situación propiamente que se conoce como cardioversión, y la otra es el intento de conservar el ritmo sinusal en un paciente que presenta sucesos recurrentes. Restituir o conservar el ritmo sinusal es muy importante, para tal cometido se puede establecer dos tipos de cardioversión: Se puede realizar mediante una descarga eléctrica o en todo caso se puede realizar con fármacos antiarrítmicos, cardioversión farmacológica. La cardioversión suele estar indicada en pacientes que presenta fibrilación auricular, por lo general de inicio reciente y también en aquello que se consideren que puede ser beneficio pasar a ritmo sinusal y por ultimo aquellos pacientes que tengan probabilidad de reversión y mantenimiento del ritmo sinusal.

Independientemente se trate de una cardioversión en sala de urgencia o de en consulta programada es importante considerar algunos aspectos importantes, puesto que la cardioversión puede aumentar el riesgo de tromboembolismo, por lo que antes de realizar cardioversión se debe establecer unas pautas específicas de seguridad 
$(3,16-18$,$) .$

\section{Cardioversión eléctrica}

La cardioversión eléctrica es un procedimiento que consiste en realizar descargas eléctricas y podemos distinguir de dos tipos interna y externa (intracardiaca y transtorácica). Durante la primera la electricidad se suministra de forma directa en el corazón mediante un dispositivo implantado para el tratamiento de arritmias y la segunda la corriente eléctrica es administrada a través de la pared del tórax por unas palas o electrodos adheridos.

Algunos aspectos importantes antes de la cardioversión es la anticoagulación correcta y un INR "índice internacional normalizado" en rango terapéutico durante las tres semanas previas caso contrario se debe descartar la presencia de un trombo en aurículas izquierda mediante la realización de un ecocardiograma transesofagico que demuestre la ausencia de trombos siendo así, aun se debe administrar una dosis de heparina (19).

"El método óptimo para la cardioversión eléctrica de fibrilación auricular incluye la selección del paciente como una técnica de cardioversión eléctrica apropiadas" (7).

Cuando se dispone de datos, se incluye también una estimación de los resultados sanitarios para grandes grupos de población. Se valoró el nivel de evidencia y la fuerza de la recomendación de una opción terapéutica particular de acuerdo con escalas predefinidas (19).

En relación con indicaciones de la cardioversión eléctrica los expertos realizaron una revisión exhaustiva de la evidencia y clases de recomendación, concluyeron un nivel de evidencia B y $\mathrm{C}$ y clase I en las siguientes situaciones; cuando la respuesta ventricular rápida no responde pronto a medidas farmacológicas en pacientes con fibrilación auricular e infarto de miocardio, hipotensión sintomática, angina, o insuficiencia cardiaca, se recomienda cardioversión eléctrica inmediata. También en síndrome de Wolff-Parkinson-White, cuando existe inestabilidad hemodinámica y los síntomas son intolerables. Y evidencia B y clase lla cuando se requiere restaurar el ritmo sinusal como parte de la estrategia del tratamiento prolongado en pacientes con fibrilación auricular. Por ultimo no es recomendado por el nivel de evidencia $\mathrm{C}$ y clase III cuando la fibrilación auricular es sintomática o recurrente, o que tienes periodo relativamente cortos de ritmo sinusal entre las recurrencias de fibrilación auricular después de varios procedimientos de cardioversión a pesar de medicación antiarrítmicos profilácticos (20).

Los factores que han sido implicados en el éxito de la cardioversión eléctrica transtorácica incluyen aquellos relacionados con la enfermedad, hábitos corporales, energía proporcionada, forma de onda eléctrica entre otros (7).

Es necesario considerar una técnica correcta para el éxito de la cardioversión eléctrica para ello es importante la posición para la colocación de los electrodos antero lateral/ anteroposterior.

Algunos estudios avalan la posición anteroposterior como más efectiva en la fibrilación auricular. Sin embargo, la mayoría han sido incapaces de demostrar una ventaja clara, por lo que se admiten ambas posiciones. En pacientes con abundante vello en 
el pecho el contacto con el electrodo-pecho puede ser malo, causando probablemente retención eléctrica entre el electrodo y la piel, pudiendo resultar en alta impedancia y ocasionalmente arcos de corriente por lo que se recomienda afeitar el área en donde se colocarán los electrodos $(20,21)$.

En la mujer, la colocación del electrodo apical en el pecho condiciona alta impedancia transtorácica, lo que reduce el flujo de corriente, por lo que se recomienda la colocación del electrodo apical en la región axilar anterior o bajo el seno (22-24).

El tamaño óptimo de los electrodos es de 8 a $12 \mathrm{~cm}$, en las últimas guías ERC 2010, recomienda utilizar electrodos autoadhesivos versus a las palas, las cuales aseguran mayor ventaja práctica.

Por lo tanto para la cardioversión en adultos, tanto los electrodos de palas metálicas de mano como electrodos de parche autoadhesivos de 8 a $12 \mathrm{~cm}$ de diámetro resultan útiles. Por otro lado, electrodos pequeños $4.3 \mathrm{~cm}$ pueden ser peligrosos (por concentrar una mayor densidad de corriente por área de superficie) y pueden causar necrosis miocárdica $(25,26)$.

\section{Cardioversión farmacológica}

La cardioversión farmacológica indicada en fibrilación auricular de inicio reciente a la que tiene una duración menor de 48 horas. Los fármacos disponibles son antiarrítmicos de la clase IC como la flecainida, propafenona, lorcainida y de la clase III la amiodarona.

Los fármacos están limitados a pacientes sin cardiopatía y su efectividad es inferior a la CV eléctrica, cuya efectividad en la actualidad con la disponibilidad de choques bifásicos es superior a $90 \%$.

La guía de la Sociedad Europea de Cardiología ESC 2016 sobre el diagnóstico y tratamiento de FA en colaboración con la European Heart Rhythm Association EACTS (19), afirma que la flecainida y la propafenona son eficaces para la cardioversión farmacológica pero su uso está restringido a pacientes sin cardiopatía estructural. La ibutilida es una alternativa, si está disponible, pero implica un riesgo de torsades de pointes. El vernakalant se puede administrar a pacientes con IC leve (NYHA I-II), incluidos los pacientes con cardiopatía isquémica, siempre que no presenten hipotensión o estenosis aórtica grave. La amiodarona se puede emplear en pacientes con IC o cardiopatía isquémica (aunque la mayoría de los estudios sobre la cardioversión de la FA excluyeron a los pacientes con IC grave). La amiodarona también ralentiza la frecuencia cardiaca en 10-12 lpm después de 8-12 h cuando se administra por vía intravenosa. Tanto la amiodarona como la flecainida parecen ser más efectivas que el sotalol para la restauración del ritmo sinusal. Para pacientes con episodios poco frecuentes de FA paroxística seleccionados, se puede indicar la autoadministración (estrategia de "pastilla en el bolsillo») de un bolo oral de flecainida (200-300 mg) o propafenona (450-600 mg) para restaurar el ritmo sinusal $(20,27-29)$.

\section{Propafenona}

Reduce la excitabilidad espontánea y la excitabilidad eléctrica en la célula ventricular. Suprime el automatismo ectópico. Prolonga el intervalo PR y el QRS, pero no alarga significativamente el intervalo QT. Es administrada por vía oral y rara vez por vía 
parenteral. Se absorbe por completo en el tubo digestivo y su vida media es de 5 a 6 horas. Se une a las proteínas plasmáticas en 95\%. Es metabolizada en el hígado y eliminada por la bilis, heces y por vía renal. Indicada en el tratamiento y prevención de extrasístole ventricular y supraventricular, incluyendo el síndrome de Wolff-Parkinson-White. Entre sus reacciones adversas causa un incremento de la disfunción ventricular que puede ocasionar IC, anomalías en la conducción y disfunción del nodo sinusal (bradicardia). Contraindicado en hipersensibilidad. Este medicamento ha resultado ser muy útil para el tratamiento de la FA y la taquicardia auricular, así como en el manejo de arritmias por reentrada que usan vías accesorias o en la reentrada nodal $(8,30-33)$.

\section{Flecainida}

La flecainida disminuye al máximo la fase del potencial de acción, así como la velocidad de conducción, aumentando la duración del complejo QRS. Se administra por vía oral y es absorbida en su totalidad en el tubo digestivo. Su vida media es de 20 horas y se elimina en $90 \%$ por vía renal. Se emplea de manera principal para el control de las arritmias supraventriculares, y en arritmias relacionadas con el síndrome de Wolff-Parkinson-White. Entre los efectos más sobresaliente puede ocasionar alteraciones visuales, cefalea, náuseas, fatiga, palpitaciones y dolor precordial y contraindicado en trastornos de la conducción AV; insuficiencia cardiaca o renal $(34,35)$.

\section{Ibutilide}

Antiarrítmicos que pertenece a la clase III cuyo mecanismo principal es bloquear el canal de potasio, causando una prolongación dependiente de dosis de intervalo QT, pero no tiene efectos significativos sobre la frecuencia cardiaca, el intervalo PR o la duración de QRS. Se administrar por vía parenteral el cual y se requiere monitorización electrocardiográfica permanente durante y después de su administración indicado para convertir la FA y el flutter auricular en ritmo sinusal. Posiblemente su efectividad en flutter auricular sea superior a la reportada con otros medicamentos, incluso en casos en los cuales la cardioversión eléctrica no obtuvo resultados $(8,36-38)$.

\section{Amiodarona}

La amiodarona fármacos de la clase III es un bloqueador de los canales de potasio y además prolongan la repolarización. Es un potente relajante del músculo liso con producción de vasodilatación periférica y coronaria. Esto puede ser por su capacidad de bloquear el paso de calcio en el músculo liso arterial coronario. Se administra por vía oral e intravenosa tiene una vida media muy prolongada de 10 a 100 días. Es metabolizado en el hígado y eliminado a través de la vesícula biliar (39).

La amiodarona es útil en la cardioversión farmacológica, potencia los resultados de la cardioversión eléctrica y ayuda a mantener el ritmo sinusal después de un episodio de fibrilación auricular; es además el menos proarrítmico de todos los antiarrítmicos y no aumenta la mortalidad en pacientes con falla cardiaca o cardiopatía isquémica, a diferencia de lo que se ha observado con otros (8).

Está indicado prevención y tratamiento de las arritmias supraventriculares, taquicardia auricular y supraventricular paroxísticas o permanentes, flúter auricular recidivante, fibrilación auricular paroxística, extrasístoles auriculares y ventriculares, y síndrome de 
preexcitación (Wolff-ParkinsonWhite) al igual que está indicado para la prevención y tratamiento de las arritmias ventriculares, incluidas la fibrilación ventricular recurrente y la taquicardia ventricular recurrente hemodinámicamente inestable $(40,41)$.

Entre las reacciones adversas los más frecuentes son toxicidad pulmonar, como fibrosis pulmonar, la exacerbación de la arritmia y lesión hepática severa. También llegan a producirse microdepósitos corneales asintomáticos en pacientes que ingieren el medicamento por más de seis meses. Contraindicada en caso de hipersensibilidad al compuesto, hepatitis aguda, alteración pulmonar intersticial y alteración de la tiroides $(28,42)$.

\section{Cardioversión eléctrica más farmacológica}

Muchas veces existe fracaso cuando se decide por un tipo de cardioversión, el fracaso de la cardioversión eléctrica inmediata o tardía se estima que ocurre aproximadamente en el $25 \%$ de los casos. Los fármacos antiarrítmicos han demostrado una pobre eficacia a largo plazo en el tratamiento de la fibrilación auricular. Se ha propuesto que la asociación de medicamentos antiarrítmicos y tratamientos no farmacológicos puede ser superior a la prescripción de un solo tratamiento. La cardioversión eléctrica de la fibrilación auricular puede ser ineficaz en varios casos (fibrilación auricular de larga duración, aurículas grandes, edad avanzada, enfermedades subyacentes, alta impedancia transtorácica): se ha demostrado que la prescripción de medicamentos antiarrítmicos antes de una descarga eléctrica puede aumentar la tasa de éxito y para reducir el requerimiento de energía. El pretratamiento con agentes clase III y IC puede ser útil para aumentar el éxito de la cardioversión eléctrica y prevenir recurrencias. La ibutilide, la amiodarona y el sotalol son los más efectivos, mientras que la eficacia de los fármacos de clase IC es controvertida $(7,19,20)$.

\section{Discusión}

La Fibrilación auricular altera la función hemodinámica del corazón, siendo un factor de riesgo de accidentes tromboembólicos, complicaciones de insuficiencias cardiacas y producir síntomas relacionados con la propia arritmia cardiaca. Restituir o conservar el ritmo sinusal es importante en el tratamiento y manejo de pacientes con fibrilación auricular para ello se establece dos tipos de cardioversión, mediante una descarga eléctrica y mediante el uso de fármacos antiarrítmicos, las cuales son válidas, sin embargo existe aspectos importantes a considerar como las indicaciones precisas y características de cada procedimiento que estableces pautas para su empleo.

La elección idónea del paciente y la técnica adecuada es significativo tanto en la cardioversión eléctrica o farmacológica. La primera puede ser de tipo urgente cuando existe inestabilidad hemodinámica en el ritmo y programada cuando no existe compromiso vital además se requiere de sedación, tromboprofilaxis de 3 semanas previas y un INR 2-3 por lo contrario la segunda es considerado cuando no existe compromiso vital y no requiere de sedación.

Referente a las indicaciones la cardioversión eléctrica es recomendada cuando la respuesta ventricular rápida no responde a medidas farmacológicas en pacientes con fibrilación auricular e infarto de miocardio, hipotensión sintomática, angina o Insuficiencia cardiaca también en preexitación (WPW) y cuando los síntomas de Fibrilación son inaceptables en pacientes hemodinámicamente estable con recurrencia temprana 
de FA postcardioversión.

En relación con cardioversión farmacológica está indicada cuando la fibrilación auricular es de corta duración, como tratamiento complementario a la cardioversión eléctrica y en casos de evitar anestesia o sedación profunda. Las complicaciones de cardioversión eléctrica suelen estar asociada a la anestesia, tromboembolismo, quemaduras cutáneas e hipotensión y la cardioversión farmacológica no presenta mayores complicaciones excepto hipersensibilidad al medicamento o la no remisión de la arritmia. Y por último el tiempo la cardioversión eléctrica es mayor a 48 horas y su efectividad es mayor por el contrario la farmacológica el tiempo de fibrilación auricular debe ser menor 48 horas y su efectividad es menor.

Por lo tanto, es posible indicar que ambos tienen sus características específicas y sus limitaciones. Al momento de actuar no hay procedimiento que pueda considerarse óptimo es necesario en algunas veces recurrir a una estrategia combinada donde el pretratamiento con antiarrítmicos de la clase III Y IC puede ser útil para aumentar el éxito de la CVE y prevenir la recurrencia de FA al igual que en una recurrencia de FA postcardioversión exitosa pueda ser útil repetir el procedimiento después de medicación antiarrítmicos profiláctico.

\section{Referencias bibliográficas}

1. John Camm A, Kirchhof P, Lip GYH, Schotten U, Savelieva I, Ernst S, et al. Guías de práctica clínica para el manejo de la fibrilación auricular. Rev Esp Cardiol. 1 de diciembre de 2010;63(12):1483.

2. Cea-Calvo L, Redón J, Lozano JV, Fernández-Pérez C, Martí-Canales JC, Llisterri JL, et al. Prevalencia de fibrilación auricular en la población española de 60 o más años de edad. Estudio PREV-ICTUS. Rev Esp Cardiol. 1 de junio de 2007;60(6):616-24.

3. Gómez-Doblas JJ, Muñiz J, Martin JJA, Rodríguez-Roca G, Lobos JM, Awamleh $P$, et al. Prevalencia de fibrilación auricular en España. Resultados del estudio OFRECE. Rev Esp Cardiol. 2014;67(4):259-69.

4. Pinto DA, Sánchez-Vallejo CA, López Pedraza A, Vergara EP, Sáenz ÓA, González F, et al. Descripción de los pacientes con fibrilación auricular no valvular que ingresan al servicio de urgencias. Rev Colomb Cardiol. 1 de julio de 2016;23(4):2706.

5. Stewart S, Hart CL, Hole DJ, McMurray JJV. Population prevalence, incidence, and predictors of atrial fibrillation in the Renfrew/Paisley study. Heart. 1 de noviembre de $2001 ; 86(5): 516-21$.

6. Alonso J. Una enfermedad prevenible: el accidente cerebrovascular en la fibrilación auricular no valvular ¿Qué lugar ocupan los nuevos anticoagulantes orales? Arch Med Interna. marzo de 2014;36(1):7-16.

7. Velázquez-Rodríguez E. La cardioversión eléctrica en fibrilación auricular. Rev Mex Cardiol. septiembre de 2012;23(3):134-50.

8. Restrepo-Jaramillo CA. Cardioversión farmacológica. Rev Colomb Cardiol. 1 de diciembre de 2016;23:52-6. 
9. Eficacia de la cardioversión programada en la fibrilación auricular. Comparación de dos esquemas de tratamiento: cardioversión eléctrica frente a cardioversión farmacológica | Revista Española de Cardiología [Internet]. [citado 6 de julio de 2019]. Disponible en: http://www.revespcardiol.org/es-eficacia-cardioversion-programada-fibrilacion-auricular--articulo-resumen-13026383

10. Orjuela Guerrero A. Cardioversión eléctrica en fibrilación auricular. Rev Colomb Cardiol. 1 de diciembre de 2016;23:57-64.

11. Restrepo-Jaramillo CA. Cardioversión farmacológica. Rev Colomb Cardiol. 1 de diciembre de 2016;23:52-6.

12. Fernández G, Maid GF, Arias AM, Maldonado S, Pizarro R, Belziti CA, et al. Prevalencia de fibrilación auricular y factores predictores de su aparición en pacientes portadores de marcapasos bicamerales. Arch Cardiol México. septiembre de 2016;86(3):214-20.

13. de Luna AB, Cygankiewicz I, Genis AB, Grande C, Viñolas X, Rodriguez E, et al. Espectro clínico y complicaciones de la fibrilación auricular. 2004;74(2):293-7.

14. Moro $C$, Hernández Madrid A. Cardioversión eléctrica para la fibrilación auricular. ¿Existen aún indicaciones tras el estudio AFFIRM? Rev Esp Cardiol. 1 de agosto de 2003;56(8):751-3.

15. Soto-Becerra R, Zafra-Tanaka JH, Goicochea-Lugo S, Alarcón-Ruiz CA, Pacheco-Barrios K, Taype-Rondan A, et al. Guía de práctica clínica para el manejo de pacientes con fibrilación auricular en el Seguro Social del Perú (EsSalud). An Fac Med. 2019;80(2):250-63.

16. Reyes Sanamé FA, Pérez Álvarez ML, Alfonso Figueredo E, Núñez Molina B, Jiménez Rodríguez K. Fibrilación auricular. Panorámica sobre un tema actualizado. Correo Científico Méd. 2018;22(4):695-718.

17. Seoane $L$, Baranchuk $A$, Conde D. Vernakalant en la reversión de la fibrilación auricular de reciente comienzo. Med B Aires. 2015;75(4):239-44.

18. Pérez-Villacastín J, Pérez Castellano N, Moreno Planas J. Epidemiología de la fibrilación auricular en España en los últimos 20 años. Rev Esp Cardiol. 2013;66(7):561-5.

19. Guía ESC 2016 sobre el diagnóstico y tratamiento de la fibrilación auricular, desarrollada en colaboración con la EACTS. Rev Esp Cardiol. 2017;70(1):50.e1-50. e84.

20. Fuster V, Rydén LE, Cannom DS, Crijns HJ, Curtis AB, Ellenbogen KA, et al. ACC/ $A H A / E S C 2006$ guidelines for the management of patients with atrial fibrillation-executive summary: a report of the American College of Cardiology/American Heart Association Task Force on Practice Guidelines and the European Society of Cardiology Committee for Practice Guidelines (Writing Committee to Revise the 2001 Guidelines for the Management of Patients with Atrial Fibrillation). Eur Heart J. 2006;27(16):1979-2030.

21. Friberg J, Gadsbøll N. Intracardiac low-energy versus transthoracic high-energy direct-current cardioversion of atrial fibrillation: a randomised comparison. Cardiol- 
ogy. 2003;99(2):72-7.

22. Pagan-Carlo LA, Spencer KT, Robertson CE, Dengler A, Birkett C, Kerber RE. Transthoracic defibrillation: importance of avoiding electrode placement directly on the female breast. J Am Coll Cardiol. febrero de 1996;27(2):449-52.

23. Atkins DL, Kerber RE. Pediatric defibrillation: current flow is improved by using «adult» electrode paddles. Pediatrics. julio de 1994;94(1):90-3.

24. Brazdzionyte J, Stanaitiene G, Ablonskyte-Dūdoniene R. [Electrical defibrillation and cardioversion]. Med Kaunas Lith. 2005;41(10):892-9.

25. Velázquez Rodríguez E, Martínez Enríquez A, Cancino Rodríguez C, Olvera Morales G, Rangel Rojo J, Arias Estrada S. Doble choque eléctrico secuencial transtorácico para la fibrilación auricular refractaria. Arch Cardiol México. 2005;75:69-80.

26. del Rey Sánchez JM, Mercader J, Ripoll E, Hernández Madrid A, González Rebollo JM, Peña Pérez G, et al. Cardioversión eléctrica externa y sistemas de cardioversión interna: evaluación prospectiva y comparativa del daño celular con troponina I. Rev Esp Cardiol. 2002;55(3):227-34.

27. January CT, Wann LS, Alpert JS, Calkins H, Cigarroa JE, Cleveland JC, et al. 2014 AHA/ACC/HRS guideline for the management of patients with atrial fibrillation: executive summary: a report of the American College of Cardiology/American Heart Association Task Force on practice guidelines and the Heart Rhythm Society. Circulation. 2014;130(23):2071-104.

28. Siebels J, Cappato R, Rüppel R, Schneider MAE, Kuck KH, Investigators CITC. Preliminary results of the Cardiac Arrest Study Hamburg (CASH). Am J Cardiol [Internet]. 1993 [citado 6 de julio de 2020];72(16). Disponible en: https://moh-it. pure.elsevier.com/en/publications/preliminary-results-of-the-cardiac-arrest-studyhamburg-cash

29. Roy D, Rowe BH, Stiell IG, Coutu B, Ip JH, Phaneuf D, et al. A randomized, controlled trial of RSD1235, a novel anti-arrhythmic agent, in the treatment of recent onset atrial fibrillation. J Am Coll Cardiol. 2004;44(12):2355-61.

30. A randomized, placebo-controlled trial of propafenone in the prophylaxis of paroxysmal supraventricular tachycardia and paroxysmal atrial fibrillation. UK Propafenone PSVT Study Group. Circulation. 1995;92(9):2550-7.

31. Boriani G, Biffi M, Capucci A, Botto GL, Broffoni T, Rubino I, et al. Oral propafenone to convert recent-onset atrial fibrillation in patients with and without underlying heart disease. A randomized, controlled trial. Ann Intern Med. 1997;126(8):621-5.

32. Khan IA. Single oral loading dose of propafenone for pharmacological cardioversion of recent-onset atrial fibrillation. J Am Coll Cardiol. 2001;37(2):542-7.

33. Alboni P, Botto GL, Baldi N, Luzi M, Russo V, Gianfranchi L, et al. Outpatient treatment of recent-onset atrial fibrillation with the «pill-in-the-pocket» approach. N Engl J Med. 2004;351(23):2384-91.

34. Sánchez Sorian RM, Chamorro Fernández CI, Ruiz Nodar JM, Chamorro Fernán$\operatorname{dez}$ AJ, Grau Jornet G, Nuñez Villota J, et al. Comparación de la eficacia y seguridad de flecainida y dronedarona como terapias antiarrítmicas para mantenimiento 
de ritmo sinusal en fibrilación auricular. Arch Cardiol México. 2018;88(3):204-11.

35. Rosenbaum M, Posse R, Sgammini H, Núnez Burgos J, Chiale PA, Pastori JD, et al. Estudio clínico multicéntrico comparativo de la flecainida y la amiodarona en el tratamiento de las arritmias ventriculares asociadas a la cardiopatia chagasica cronica. Arch Inst Cardiol Méx. 1987;57(4):325-30.

36. Naccarelli GV, Lee KS, Gibson JK, VanderLugt J. Electrophysiology and pharmacology of ibutilide. Am J Cardiol. 1996;78(8A):12-6.

37. Stambler BS, Wood MA, Ellenbogen KA, Perry KT, Wakefield LK, VanderLugt JT. Efficacy and safety of repeated intravenous doses of ibutilide for rapid conversion of atrial flutter or fibrillation. Ibutilide Repeat Dose Study Investigators. Circulation. 1996;94(7):1613-21.

38. Oral H, Souza JJ, Michaud GF, Knight BP, Goyal R, Strickberger SA, et al. Facilitating transthoracic cardioversion of atrial fibrillation with ibutilide pretreatment. $\mathrm{N}$ Engl J Med. 1999;340(24):1849-54.

39. Chevalier P, Durand-Dubief A, Burri H, Cucherat M, Kirkorian G, Touboul P. Amiodarone versus placebo and class Ic drugs for cardioversion of recent-onset atrial fibrillation: a meta-analysis. J Am Coll Cardiol. 2003;41(2):255-62.

40. Goldschlager N, Epstein AE, Naccarelli GV, Olshansky B, Singh B, Collard HR, et al. A practical guide for clinicians who treat patients with amiodarone: 2007. Heart Rhythm. 2007;4(9):1250-9.

41. Daoud EG, Strickberger SA, Man KC, Goyal R, Deeb GM, Bolling SF, et al. Preoperative amiodarone as prophylaxis against atrial fibrillation after heart surgery. $\mathrm{N}$ Engl J Med. 1997;337(25):1785-91.

42. Giri S, White CM, Dunn AB, Felton K, Freeman-Bosco L, Reddy P, et al. Oral amiodarone for prevention of atrial fibrillation after open heart surgery, the Atrial Fibrillation Suppression Trial (AFIST): a randomised placebo-controlled trial. Lancet Lond Engl. 2001;357(9259):830-6. 